\title{
PERFORMANCE INDICES OF TWO DIFFERENT REPEATED SPRINT TESTS PROTOCOLS IN OVERWEIGHT CHILDREN
}

\author{
Y. Meckel ${ }^{1}$, D. Nemet ${ }^{2}$, S. Lougassi ${ }^{1}$, A. Eliakim ${ }^{1,2}$ \\ ${ }^{1}$ Zinman College of Physical Education and Sport Sciences, \\ Wingate Institute, Israel \\ ${ }^{2}$ Child Health and Sport Center, Pediatric Department, Meir Medical \\ Center, Sackler School of Medicine, Tel-Aviv University, Israel
}

\begin{abstract}
We determined the relationship between aerobic fitness and a short and long repeated sprint test (RST) in pre pubertal overweight children $(10.5 \pm 1.5 \mathrm{yrs})$. Aerobic fitness was evaluated by the $20 \mathrm{~m}$ shuttle run. Two RST protocols with identical total running distance were performed to determine anaerobic capacities at random order $(12 \times 20 \mathrm{~m}$ run departing every $20 \mathrm{~s}$ and $6 \times 40 \mathrm{~m}$ run departing every $40 \mathrm{~s})$. Performance decrement (PD) and total sprint time (TS) were significantly higher in the $12 \times 20 \mathrm{~m}$ compared to the $6 \times 40 \mathrm{~m}$ protocol. Significant negative correlations were found between the aerobic fitness and TS $(r=-0.767)$ and the fastest sprint time $(r=-0.738)$ of the $12 \times 20 \mathrm{~m}$ protocol. Similarly, significant negative correlations were found between the aerobic fitness and TS $(\mathrm{r}=-0.803)$ and the fastest sprint time $(\mathrm{r}=-0.787)$ of the $6 \times 40 \mathrm{~m}$ protocol. There were no significant correlations between PD in both RST's and aerobic fitness. Performance of high number of short repetitions with very short recovery time is more difficult for overweight children than fewer longer repetitions with longer recovery time. Aerobic fitness plays an important role in intense intermittent activity, but not in PD from intermittent activity in obese children.
\end{abstract}

Key words: aerobic, anaerobic fitness, childhood obesity, repeated sprint test 


\section{INTRODUCTION}

Children usually engage in spontaneous short exercise bursts separated by brief rest periods [2]. The ability of children to sustain intensity during repeated sprints was previously examined [24, 25, 33] using a wide range of age groups (9-17 yrs.) and different protocols of a repeated sprint test (RST). Compared to adults, small decrease in running performance was found in children [10]. Differences were significantly greater when the recovery intervals were short $(15 \mathrm{sec})$ as opposed to long $(180 \mathrm{sec})$. In addition, prepubertal males $(9.6 \pm 0.7$ yrs.) needed less recovery time to sustain peak power output compared to adolescent males $(15.0 \pm 0.7 \mathrm{yrs})$ or young adults $(20.4 \pm 0.8 \mathrm{yrs}$.) during repeated cycling sprints [24]. Consistent with that, pre-pubertal children repeated their initial performance faster following highintensity exercise compared to young adults $[17,26]$.

Aerobic fitness was found previously as a prerequisite for anaerobic performance during repeated sprints [30]. Despite that, correlation analyses between $\mathrm{VO}_{2}$ max and performance indices of RST were found inconsistent, and only few studies reported significant correlations between the two tests [e.g., 10, 20]. These relationships are especially relevant to children, due to their characteristic intermittent activity patterns. Testing the relationship between aerobic fitness and RST performance indices is even more challenging in overweight children. This group is particularly important due to the increased prevalence of childhood obesity in Westernized societies [27], and since both aerobic and anaerobic capacities are reduced in obese children [11, 19, 31]. Physical activity patterns of obese children are also characterized by brief exercise bouts, performed at different intensities and separated by different rest intervals [9, 21]. Thus, assessment of RST indices and their relationship to aerobic fitness in this population is important. Surprisingly, to the best of our knowledge, these relationships were not studied in obese children.

There are different protocols of RSTs; some use short sprint intervals, while others use long sprint intervals. The aim of the present study was to compare between short (i.e., $12 \times 20 \mathrm{~m}$ ), and long (i.e., $6 \times 40 \mathrm{~m}) \mathrm{RST}$ protocols, and to determined the relationship between the short and the long RST and aerobic fitness (measured by the $20 \mathrm{~m}$ shuttle run test) in pre- and early-pubertal overweight children. Since patterns of physical activity among overweight children are usually characterized by short, rather than long, activity intervals, we 
hypothesized that the performance decrement will be lower in the short compared to the long RST protocol, and that the shorter RST protocol will better correlate with aerobic fitness.

\section{MATERIAL AND METHODS}

Fourteen children $($ males $=6$, females $=8 ; 10.5 \pm 1.5 \mathrm{yrs}$.) volunteered to participated in the study. All children participated in a weight reduction program in the Child Health and Sport Center at the Pediatric Department of Meir Medical Center. The study was approved by the Institution's ethical committee. All the children were assessed for obesity status and Tanner stage [18]. The testing procedure was explained to the children and to their parents, and a written informed consent was obtained from both.

\section{Procedure}

All participants performed three tests, separated by a week from each other, in random order. The three tests were an aerobic power test, a short interval RST and a long interval RST. Before each test, the children participated in a special habituation session.

\section{Performance tests}

Aerobic Power Test - Twenty-Meter Shuttle Run Test

The 20-meter shuttle run test is a field test that has been shown to be a reliable and valid indicator [28] of aerobic power in various populations [22]. The main reason for the use of this test in the present study to evaluate aerobic fitness was its back and forward run which characterizes children's voluntary activity patterns. It also eliminates the need to use laboratory equipment such as a motor driven treadmill and a face mask, which are unfamiliar and uncomfortable to children and especially to obese children. The test consists of shuttle running at increasing speeds between two markers placed $20 \mathrm{~m}$ apart. A portable compact disc (Sony CFD-V7) dictated the pace of the test by emitting tones at appropriate intervals. The children were required to be at one end of the $20 \mathrm{~m}$ course at the signal. A starting speed of $8.5 \mathrm{~km} /$ hour was maintained for one minute, and thereafter the speed was increased every minute by $0.5 \mathrm{~km} /$ hour. The test was terminated when the child withdrew voluntarily from the exercise, or failed to arrive within 3 meters of the end line on two consecutive tones. The aerobic fitness of 
each participant was calculated as the total distance achieved during the test.

\section{Repeated sprint tests}

Two protocols of the RST were performed by the participants. Each protocol included a series of maximal runs with short rest periods between runs. The two protocols consisted of the following:

1. A short interval RST - Twelve X 20m runs starting every $20 \mathrm{~s}$.

2. A long interval RST - Six X 40m runs starting every 40s.

A $20 \mathrm{~m}$ and a $40 \mathrm{~m}$ all-out sprint were performed following the warm-up of the $12 \times 20 \mathrm{~m}$ and the $6 \times 40 \mathrm{~m}$ protocols, respectively, by each participant. The time for each sprint was used as the criterion score for the subsequent RST. In the first sprint of each RST, participants were required to achieve at least $95 \%$ of their criterion score. If $95 \%$ of the criterion score was not achieved, the participant was required to start the RST again. None of the participants was required to restart the RST due to a slow initial sprint.

A photoelectric cell timing system (Alge-Timing Electronic, Vienna, Austria) linked to a digital chronoscope was used to record each sprint and rest interval time with an accuracy of $0.001 \mathrm{~s}$. During the recovery period between sprints, participants tapered down from the sprint they had just completed and slowly walked back to the next starting point. Two sets of timing gates were used, working in opposite directions, to allow participants to start the next run from the end-point of the preceding sprint, thus eliminating the need to hurry back to the same starting point. A standing start, with the front foot placed $30 \mathrm{~cm}$ behind the timing lights, was used for all sprints. Timing was initiated when the participants broke the light beam. An experimenter was placed at each end of the track to give strong verbal encouragement to each participant at each sprint. Participants were instructed prior to the test to produce maximal effort for each sprint and to avoid pacing themselves.

The three measures of each RST $(12 \times 20 \mathrm{~m}$ and $6 \times 40 \mathrm{~m})$ were the fastest $20 \mathrm{~m}$ or $40 \mathrm{~m}$ sprint time (FS), the total sprint time (TS) of the 12 or 6 sprints, and the performance decrement (PD) during each test. TS was calculated as the sum of all sprints times of each test. PD was used as an indication of fatigue and was calculated by dividing the sum of the sprinting times for each of the 6 or 12 sprints by the best possible total score multiplied by 100 [13]. The best possible total score was calculated as the fastest $40 \mathrm{~m}$ or $20 \mathrm{~m}$ sprint time multiplied 
by 6 or 12, respectively. The test-retest reliability of the RST is 0.942 for total running time, and 0.75 for performance decrement [13].

Heart rate was measured using a Polar heart rate monitor (Polar Accurex Plus, Polar Electro, Woodbury, NY) immediately after completion of each run in both RSTs. Rate of perceived exertion (RPE) was determined using the modified Borg scale [8] at the end of each RST.

\section{Statistical analyses}

Paired t-test was used for comparing differences (in fastest and total sprint time, performance decrement, heart rate, RPE, etc.) between the two different RST protocols. Pearson correlations were computed between the calculated distance during the shuttle run aerobic test and performance indices of the two different RSTs. Data are presented as mean \pm SD. Significance level was set at $\mathrm{p}<0.05$.

\section{RESULTS}

Anthropometric characteristics of the study participants and the results of the $20 \mathrm{~m}$ shuttle run aerobic test are provided in Table 1.

Table 1. Anthropometric measures and total distance (Mean \pm SD) during the $20 \mathrm{~m}$ shuttle run of the study participants $(\mathrm{N}=14)$.

\begin{tabular}{|l|c|}
\hline Parameters & Mean \pm SD \\
\hline Age (yrs) & $10.5 \pm 1.5$ \\
\hline Pubertal Stage (Tanner) & $1.2 \pm 0.4$ \\
\hline Body Height $(\mathrm{m})$ & $143.5 \pm 7.9$ \\
\hline Body Weight $(\mathrm{kg})$ & $52.5 \pm 10.0$ \\
\hline BMI $\left(\mathrm{kg} / \mathrm{m}^{2}\right)$ & $25.1 \pm 4.7$ \\
\hline BMI percentile $(\%)$ & $96.4 \pm 1.9$ \\
\hline Distance-20m shuttle run $(\mathrm{m})$ & $468.6 \pm 107.4$ \\
\hline
\end{tabular}

Performance indices of the two different RST protocols are given in Table 2. 
Table 2. Performances indices (Mean $\pm \mathrm{SD}$ ) and protocol characteristics of the two RSTs $(\mathrm{N}=14)$.

\begin{tabular}{|l|c|c|}
\hline Indices & $\mathbf{6} \times \mathbf{4 0 m}$ & $\mathbf{1 2 \times 2 0 m}$ \\
\hline Fastest Sprint (sec) & $9.01 \pm 1.35$ & $4.71 \pm 0.65^{*}$ \\
\hline Total Sprint Time (sec) & $56.04 \pm 8.71$ & $60.30 \pm 9.05^{*}$ \\
\hline Performance Decrement (\%) & $3.62 \pm 1.62$ & $6.56 \pm 3.62^{*}$ \\
\hline Total Run Distance (m) & 240 & 240 \\
\hline Total Rest Time (sec) & 200 & $220^{*}$ \\
\hline Total Practice Time (sec) & $256.0 \pm 8.7$ & $280.3 \pm 9.1^{*}$ \\
\hline Maximal Heart Rate (beats/min) & $186.4 \pm 11.1$ & $192.2 \pm 8.3$ \\
\hline RPE Score & $3.6 \pm 1.3$ & $5.1 \pm 1.5^{*}$ \\
\hline
\end{tabular}

* $\mathrm{p}<0.05$ for between-test differences

The performance decrement, total sprint time, total rest time, and total practice time were significantly higher in the $12 \times 20 \mathrm{~m}$ protocol than in the $6 \times 40 \mathrm{~m}$ protocol. The fastest sprint time was significantly lower in the $12 \times 20 \mathrm{~m}$ protocol than in the $6 \times 40 \mathrm{~m}$ protocol. RPE scores were significantly higher in the $12 \times 20 \mathrm{~m}$ protocol than in the $6 \times 40 \mathrm{~m}$ protocol.

The correlations between the $20 \mathrm{~m}$ shuttle run aerobic test and performance indices of the two RSTs are summarized in Table 3 and Figure 1.

Table 3. Relationships between $20 \mathrm{~m}$ distance shuttle run and performance indices in the two RSTs. Data presented as $r$ values.

\begin{tabular}{|l|l|c|}
\hline RST Protocol & Performance Indices & 20m Shuttle Run \\
\hline $6 \times 40 \mathrm{~m}$ RST & Fastest Sprint Time $(\mathrm{sec})$ & $-0.787^{*}$ \\
\cline { 2 - 3 } & Total Sprint Time $(\mathrm{sec})$ & $-0.803^{*}$ \\
\cline { 2 - 3 } & Performance Decrement $(\%)$ & -0.344 \\
\hline \multirow{3}{*}{$12 \times 20 \mathrm{~m}$} & Fastest Sprint Time $(\mathrm{sec})$ & $-0.738^{*}$ \\
\cline { 2 - 3 } & Total Sprint Time $(\mathrm{sec})$ & $-0.767^{*}$ \\
\cline { 2 - 3 } & Performance Decrement (\%) & -0.326 \\
\hline
\end{tabular}

* Significant correlation at $\mathrm{p}<0.05$ 

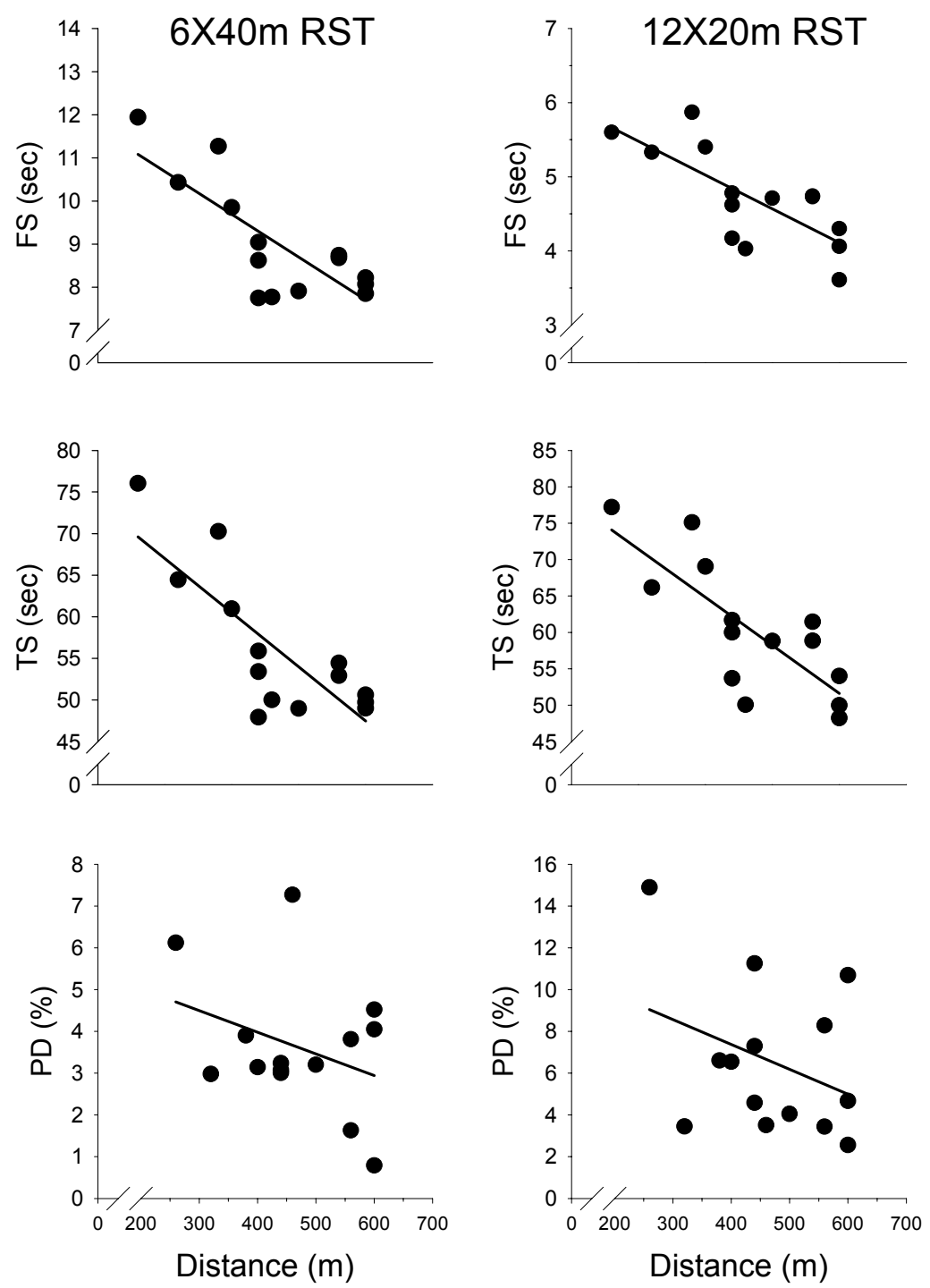

Figure 1. Relationships between the total distance in the $20 \mathrm{~m}$ shuttle run and performance indices in the long (left panel) and short (right panel) RST. 
Significant negative correlations were found between the $20 \mathrm{~m}$ shuttle run aerobic test and the total sprint time $(\mathrm{r}=-0.767)$ and the fastest sprint time $(\mathrm{r}=-0.738)$ of the short RST $(12 \times 20 \mathrm{~m})$. Similarly, significant negative correlations were found between the $20 \mathrm{~m}$ shuttle run aerobic test and the total sprint time $(\mathrm{r}=-0.803)$ and the fastest sprint time $(\mathrm{r}=-0.787)$ of the long RST $(6 \times 40 \mathrm{~m})$. There were no significant correlations between the performance decrement in the long or the short RST and the $20 \mathrm{~m}$ shuttle run aerobic test $(\mathrm{r}=-0.344$ and $\mathrm{r}=-0.326$ for the long and short RST, respectively).

\section{DISCUSSION}

The present study examined the relationship between performance indices of short (i.e., $12 \times 20 \mathrm{~m}$ ) and long (i.e., $6 \times 40 \mathrm{~m}$ ) interval RSTs, and aerobic fitness (measured by the $20 \mathrm{~m}$ shuttle run test) in pre- and early- pubertal overweight children. Despite the identical total running distance of the two RSTs, the TS, PD and total practice time were significantly higher in the short compared to the long RST. The RPE score was significantly higher in the short compared to the long RST. In addition, the maximal heart rate was higher (although not significantly) in the short compared to the long RST. These results indicate that performance of higher number of short repetitions with short recovery time was more difficult for overweight children than fewer but longer repetitions with relatively long recovery time. It seems that the physiological load of intermittent activity in overweight children depends on the specific variables, even if the total work of two different intermittent sessions is identical. These variables refer primarily to the number and duration of repetitions, and to the duration of the recovery periods.

Although we are unaware of studies that examined RST performance in overweight or obese children, several studies compared RST performances of normal weight children to adults [e.g., 17, 24, 25, 26]. Ratel et al. [24] found that prepubescent boys ( $9.6 \pm 0.7$ yrs.) sustained their peak power output during ten $10 \mathrm{sec}$ sprint exercises separated by $30 \mathrm{sec}$ recovery intervals, while pubertal boys $(15.0 \pm 0.7$ yrs.) and young adult males $(20.4 \pm 0.8$ yrs) needed 5 min recovery intervals. In another study, Ratel et al. [25] found that running performance decreased less in boys $(11.7 \pm 0.5 \mathrm{yrs})$ compared to men $(22.1 \pm 2.9$ yrs.) during ten repeated $10 \mathrm{sec}$ treadmill sprints separated 
by $15 \mathrm{sec}$ recovery intervals. Three-minute recovery periods were sufficient for the boys to repeat short running sprints without substantial fatigue and a consequent performance decrement. In contrast, although the young men were able to modify their running style to maintain running velocity during the test, power and force output decreased significantly. Taylor et al. [29] suggested that the faster recovery in boys was due to their lower glycolitic activity and higher muscle oxidative capacity, allowing a faster re-synthesis of energy stores.

In the present study we tested a group of pre and early pubertal overweight children during two forms of RST. It was found that the PD was significantly higher in the short RST protocol $(6.6 \%$ compared to $3.6 \%$ in the short versus the long RST protocol, $\mathrm{p}<0.05$, Table 2). The decrement in running speed during the RST could be the result of a reduction in energy supply and/or acidosis of the muscle cell. The stores of phosphocreatine ( $\mathrm{PCr})$, which are essential to the reconstitution of short-term power output [7], could have been only partially replenished following each sprint, since a complete phosphagen recovery in children requires more than the 20 (short RST) or $40 \mathrm{sec}$ (long RST) rest period that was used in the present study [7, 15]. However, the higher number of repetitions and the shorter recovery time probably made the short RST protocol more difficult compared to the long RST protocol. Yet the average sprint time in the long RST protocol was about twice that of the average sprint time of the short protocol. It is possible, therefore, that lactic acid accumulation and a feeling of local muscle fatigue would be greater following the long RST. Lactate levels were not measured in the present study; however, it is well known that exercise-induced lactic acid accumulation is markedly lower in pre-pubertal children compared to latepubertal children and adults during repeated sprint exercise, due to their lower glycolytic energy turnover $[12,16]$. This may explain the relative fast recovery and the lower PD of the overweight pre-pubertal children during the long RST in the present study. It is possible that if more sprints had been performed in the long RST, then fatigue and a significant decrease in performance would occur. Therefore, it seems that the length of the recovery time is a more significant factor than the length of the sprints during repeated activity in pre-pubertal overweight children. The results emphasize the need for selection of an appropriate RST protocol for performance assessment, one that will 
match the activity characteristics and patterns of the specific population.

The PD values found in the present study are similar to PD values that were found among adult athletes in other studies [e.g., 4-6\%; 10, $13,32]$. This supports the assumption that the recovery process among children is faster than in adults [7, 29], and that even overweight children are able to maintain exercise intensity relatively well throughout repeated sprints, in spite of their excessive weight and low aerobic capacity. In contrast, PD was higher in overweight children, indicating greater fatigue, compared to that of non-obese children, who were able to maintain their peak power with no significant reduction during RST [24, 25]. In addition, it should be emphasized that $\mathrm{PD}$ is determined relative to the personal results, which were far worse in the obese children of the present study compared to results in studies of non-obese children or adult athletes [9, 21, 31].

The present study also examined the relationship between the aerobic capacity (measured by the running distance of the $20 \mathrm{~m}$ shuttle run aerobic test) and the performance indices of the short and long RSTs. In contrast to our hypothesis, significant strong negative correlations were found between the aerobic capacity and the TS or the FT of both RSTs (see Table 3). Although PCr resynthesis appears to be controlled by the rate of oxidative metabolism within the muscle [29], previous studies have found only moderate correlations between $\mathrm{VO}_{2}$ max and performance indices of RSTs [10, 14, 20,]. Glaister et al. [14] found moderate correlations between $\mathrm{VO}_{2}$ max and power output regardless of recovery duration $(10$ or $30 \mathrm{sec})$ during repeated sprints. However, the relationships were stronger when power output data were averaged over all the sprints, supporting previous reports of moderate negative correlations between $\mathrm{VO}_{2}$ max and total intermittent sprint time $[1,10]$. These results are consistent with the present findings of strong negative correlations between aerobic capacity and the TS of both RSTs $(r=-0.803$ and $r=-0.767$, for the long and short RST, respectively; $\mathrm{p}<0.05$ ). Moreover, aerobic capacity was significantly correlated to the FT of the participants in both RSTs as well $(r=-0.787$ and $r=-0.738$, for the long and short RST, respectively; $\mathrm{p}<0.05)$. These findings are in contrast to previous reports indicating that the aerobic components of a single short sprint $(\leq 10 \mathrm{sec})$ are very small $(<10 \%)$ in adults subjects $[5,23]$. Therefore, the results of the present study may suggest that oxidative metabolism plays an important role, serving as an energy source even during a 
single sprint, in overweight children [16]. The magnitude of the correlation between FT and TS of both RSTs with aerobic capacity was unaffected by the differences in sprint length, sprint number, and recovery times between the long and short RST. This suggests that in overweight children other factors play an important role in these relationships (e.g., individual speed or anaerobic capacity). It also emphasizes again the need to find a protocol that will best fit the activities and the relationship between aerobic and anaerobic capacities of overweight children.

No significant correlations were found between the aerobic capacity and the PD of both RSTs in the present study (Table 3). This was somewhat unexpected in light of the strong correlations between aerobic capacity and the TS in both RSTs. However, while oxygen availability may influence fatigue during repeated sprints $[3,4]$, there is no substantial evidence of such link [1, 6, 10, 32]. Therefore, it is not surprising that correlations between $\mathrm{VO}_{2}$ max and PD during RSTs have been inconsistent, and while some studies found significant correlations between the two [10], others failed to do so [32]. It is also possible that the magnitude of this association may be largely determined by the specific RST protocol. It could be hypothesized that the relatively short sprints in both RSTs in the present study resulted in only a partial depletion of the PCr stores. Therefore, the ability to re-synthesize these stores had no effect on the aerobic energy system. In contrast, in RST protocols involving longer (15-90 sec) high intensity sprints with longer recovery periods $(1.5-7 \mathrm{~min})$ and possibly greater depletion of $\mathrm{PCr}$, the aerobic system had been shown to display significant correlations with fatigue and with total sprint time $[10,20]$. The lack of correlation between aerobic capacity and performance decrement may also be explained by the heterogeneity of running skills and running efficiency among the pre-pubertal overweight children. Reduced running efficiency affects performance mainly during all out sprints, as was the case in both RSTs of the present study.

In summary, the physiological load of intermittent activity in prepubertal overweight children is significantly dependent on its specific variables even if the total work of two different intermittent sessions is identical. Specifically, performance of a high number of short repetitions with very short recovery time seems to be a more difficult task for overweight children than the performance of fewer longer repetitions with a longer recovery time. The significant correlations of 
TT and FS of both RST protocols with aerobic capacity suggest that oxidative metabolism plays an important role in intense intermittent activity, even during a single all-out sprint in pre-pubertal overweight children. The lack of significant correlation between aerobic capacity and performance decrement during intermittent activity in overweight children suggests that factors other than aerobic fitness may be important for intensity maintenance during intermittent exercise in this population. Since activities of obese children are characterized by repeated short exercise bursts, performance of the traditional fitness tests like maximal oxygen consumption (10-12 $\mathrm{min}$ ) or the Wingate anaerobic test $(30 \mathrm{sec})$ does not represent their usual activity pattern, and RST may serve as another good alternative. More research is needed to select the best RST protocol for this population.

\section{REFERENCES}

1. Aziz A. R., Chia M., Teh K. C. (2000) The relationship between maximal oxygen uptake and repeated sprint performance indices in field hockey and soccer players. J. Sports Med. Phys. Fitness. 40: 195-200

2. Bailey R. C., Olson J., Pepper S. L., Barstow T. J., Cooper D. M. (1995) The level and tempo of children's physical activity: an observation study. Med. Sci. Sports Exerc. 27: 1033-1041

3. Balsom P. D., Ekblom B., Sjodin B. (1994) Enhanced oxygen availability during high intensity intermittent exercise decreases anaerobic metabolite concentrations in blood. Acta Physiol. Scand. 150:455-456

4. Balsom P. D., Gaitanos G. C., Ekblom B., Sjodin B. (1994) Reduced oxygen availability during high intensity intermittent exercise impairs performance. Acta Physiol. Scand. 152: 279-285

5. Bangsbo J., Krustrup P., Gonzalez-Alonso J., Saltin B. (2001) ATP production and efficiency of human skeletal muscle during intense exercise: effect of previous exercise. Am. J. Physiol. Endocrinol. Metab. 280: 956-964

6. Bishop D., Lawrence S., Spencer M. (2003) Predictors of repeatedsprint ability in elite female hockey players. J. Sci. Med. Sport. 6: 199-209

7. Bogdanis G. C., Nevill M. E., Boobis L. H., Lakomy H. K. A. (1996) Contribution of phosphocreatine and aerobic metabolism to energy supply during repeated sprint exercise. J. Appl. Physiol. 80: 876-884 
8. Borg G. A. (1982) Psychophysical bases of perceived exertion. Med. Sci. Sports Exerc. 14: 377-382

9. Cooper A. R., Page A., Fox K. R., Misson J. (2000) Physical activity patterns in normal, overweight and obese individuals using minuteby-minute accelerometry. Eur. J. Clin. Nutr. 54: 887-894

10. Dawson B. T., Fizsimons M., Ward D. (1993) The relationship of repeated sprint ability to aerobic power and performance measures of anaerobic work capacity and power. Aus. J. Sci. Med. Sport. 25: 8892

11. Eliakim A., Nemet D., Zaldivar F., McMurray R. G., Culler F. L., Galassetti P., Cooper D.M. (2006) Reduced exercise-associated response of the GH-IGF-I axis and catecholamines in obese children and adolescents. J. Appl. Physiol. 100: 1630-1637

12. Eriksson O., Saltin B. (1974) Muscle metabolism during exercise in boys aged 11 to 16 years compared to adults. Acta Paediatr. Belg. 28: 257-265

13. Fitzsimons M., Dawson B. T., Ward D., Wilkinson A. (1993) Cycling and running tests of repeated sprint ability. Aus. J. Sci. Med. Sport. 25: $82-87$

14. Glaister M., Stone M. H., Stewart A. M., Hughes M. G., Moir G. (2006) Aerobic and anaerobic correlates of multiple sprint cycling performance. J. Strength Con. Res. 20: 792-798

15. Harris R. C., Edwards R. H. T., Hultman E., Nordesjo L. O., Nylind B., Sahlin K. (1976) The time course of phosphocreatine resynthesis during recovery of the quadriceps muscle in man. Pflugers Arch. 376: $137-142$

16. Hebestreit H., Meyer F., Htay H., Heigenhauser G. J., Bar-Or O. (1996) Plasma metabolites, volume and electrolytes following $30 \mathrm{~s}$ high-intensity exercise in boys and men. Eur. J. Appl. Physiol. 72: 563-569

17. Hebestreit H., Mimura K., Bar-Or O. (1993) Recovery of muscle power after high-intensity short-term exercise: comparing boys and men. J. Appl. Physiol. 74: 2875-2880

18. Kuczmarski R. J., Ogden C. L., Guo S. S., Grummer-Strawn L. M., Flegal K. M., Mei Z., Wei R., Curtin L. R., Roche A. F., Johnson C. L. (2002) 2000 CDC growth charts for the United States: methods and development. Vital Health Stat. 246: 1-190

19. Marinov B., Kostianev S., Turnovska T. (2000) Ventilatory efficiency and rate of perceived exertion in obese and non-obese children performing standardized exercise. Clin. Physiol. Funct. Imag. 22: $254-260$ 
20. McMahon S., Wenger H.A. (1998) The relationship between aerobic fitness and both power output and subsequent recovery during maximal intermittent exercise. J. Sci. Med. Sport. 1: 219-227

21. Page A., Cooper A. R., Stamatakis E., Foster L. J., Crowne E. C., Sabin M., Shield J. P. (2005) Physical activity patterns in nonobese and obese children assessed using minute-by-minute accelerometry. Int. J. Obes. 29: 1070-1076

22. Paliczka V. J., Nichols A. K., Boreham C. A. G. (1987) A multi-stage shuttle run as a predictor of running performance and maximal oxygen uptake in adults. Br. J. Sports Med. 21: 163-165

23. Parolin M. L., Chesley A., Matsos M. P., Striet L. L., Jones N. L., Heigenhauser G. J. F. (1999) Regulation of skeletal muscle glycogen phosphorylase and PDH during maximal intermittent exercise. Am. J. Physiol. 277: 890-900

24. Ratel S., Bedu M., Hennegrave A., Dore E., Duche P. (2002) Effects of age and recovery duration on peak power output during repeated cycling sprints. Int. J. Sports. Med. 23: 397-402

25. Ratel S., Williams C. A., Oliver J., Armstrong N. (2006) Effects of age and recovery duration on performance during multiple treadmill sprints. Int. J. Sports Med. 27: 1-8

26. Soares J. M. C., Mota P., Duarte J. A., Appell H. J. (1996) Children are less susceptible to exercise-induced muscle damage than adults: a preliminary investigation. Pediatr. Exerc. Sci. 8: 361-367

27. Spiser P. W., Rudolf M. C., Anhalt H., Camacho-Hubner C., Chiarelli F., Eliakim A., Freemark M., Gruters A., Hershkovitz E., Iughetti L., Krude H., Latzer Y., Lustig R. H., Hirsch Pescovitz O., Pinhas-Hamiel O., Rogol A. D., Shalitin S., Sultan C., Stein D., Vardi P., Werther G. A., Zadik Z., Zuckerman-Levin N. (2005) Childhood obesity. J. Clin. Endocrinol. Metab. 90: 1871-1887

28. St Clair Gibson A., Broomhead S., Lambert M. I., Hawley J. A. (1998) Prediction of maximal oxygen uptake from a 20m shuttle run as measured directly in runners and squash players. J. Sports. Sci. 16: 331-335

29. Taylor D. J., Kemp G. J., Thompson C. H., Radda G. K. (1997) Aggeing: effects on oxidative function of skeletal muscle in vivo. Mol. Cell. Biochem. 174: 321-324

30. Tomlin D. L., Wenger H. A. (2001) The relationship between aerobic fitness and recovery from high intensity intermittent exercise. Sports Med. 31: 1-11

31. Unnithan V. B., Nevill A., Lange G., Eppel J., Fischer M., Hebestreit H. (2006) Applicability of an allometric power equation to children, 
adolescents and young adults of extreme body size. J. Sports Med. Phys. Fitness. 46: 202-208

32. Wadley G., Le RossIgnol P. (1993) The relationship between repeated sprint ability and the aerobic and anaerobic energy systems. J. Sci. Med. Sport. 1: 100-110

33. Yanagiya T., Kanehisa H., Kouzaki M., Kawakami Y., Fukunaga T. (2002) Effect of gender on mechanical power output during repeated bouts of maximal running in trained teenagers. Int. J. Sports Med. 24: 304-310

\section{Correspondence to:}

Yoav Meckel

Life Science Department

The Zinman College for Physical Education and Sport Sciences

Wingate Institute, 42902

Israel

E-mail:meckel@wincol.ac.il 